\title{
JACKSON NETWORKS WITH UNLIMITED SUPPLY OF WORK
}

\author{
GIDEON WEISS, * The University of Haifa
}

\begin{abstract}
We consider a Jackson network in which some of the nodes have an infinite supply of work: when all the customers queued at such a node have departed, the node will process a customer from this supply. Such nodes will be processing jobs all the time, so they will be fully utilized and experience a traffic intensity of 1 . We calculate flow rates for such networks, obtain conditions for stability, and investigate the stationary distributions. Standard nodes in this network continue to have product-form distributions, while nodes with an infinite supply of work have geometric marginal distributions and Poisson inflows and outflows, but their joint distribution is not of product form.
\end{abstract}

Keywords: Queue; Jackson network; infinite virtual buffer

2000 Mathematics Subject Classification: Primary 60K25; 90B22; 68M20; 90B15

Consider a Jackson network [5] with nodes $i$, service rates $\mu_{i}$, exogenous input rates $\alpha_{i}$, and routing probabilities $P_{i j}, i, j=1, \ldots, I, j \neq i$. Denote by $Q_{i}(t), t>0$, the number of items in node $i$ at time $t$. In addition, assume that a subset of the nodes $E \subseteq\{1, \ldots, I\}$ have an infinite supply of work, by which we mean the following. For $i \in E$, when $Q_{i}(t)=0$, a new item is picked from an infinite supply of items and is processed by the node at the rate $\mu_{i}$. Upon completion of processing, it is routed according to $P_{i j}$. At each of the nodes $i \in E$, items that arrive at the node have preemptive priority over items from the infinite supply. After its initial processing, an item from the infinite supply is treated like any other item.

We believe that such Jackson networks with an infinite supply of work are a useful and realistic model for some situations; for example, consider a communications network, where each node is transmitting messages originating at this node, with an unlimited supply of material to transmit. In addition, each node also serves to transmit messages that are in transit between other nodes. Assume that each node gives preemptive priority to messages in transit over its own messages. When only messages in transit are counted as congestion, this is exactly our system. A particular computer communication system that works in this way is a MAN (metropolitan area network) Ethernet RPR (resilient packet ring), in which ring traffic has priority over the traffic generated at nodes.

The idea of an infinite supply (or backlog) of lower-priority work in a system has been used frequently, e.g. in [3] and [6]. Jackson networks with an infinite supply of work are a special case of multiclass queueing networks with virtual infinite buffers, introduced in [2], [8], [9], and [10].

Recall that all service and interarrival times in a Jackson network are independent and exponentially distributed. Also, assume that the routing matrix $\boldsymbol{P}$ has spectral radius less than 1 ,

Received 16 November 2004; revision received 21 February 2005.

* Postal address: Department of Statistics, The University of Haifa, Mount Carmel, 31905, Israel.

Email address: gweiss@ @stat.haifa.ac.il

Supported in part by Israel Science Foundation grant number 249/02. 
so that, with probability 1 , every item leaves the system after a finite number of processing steps. Let $\bar{E}=\{i: i \notin E\}$. We will use $\lambda, \mu$, and $\boldsymbol{\alpha}$ to denote vectors, and $E, \bar{E}$, and $E \bar{E}$, as subscripts, to denote subvectors or submatrices.

Let $\lambda_{i}$ denote the rate at which items arrive into node $i$, counting exogenous input or routeing from other nodes. In equilibrium, the rate at which items depart from node $i$ is $\lambda_{i}, i \in \bar{E}$, and $\mu_{i}, i \in E$. Hence, the traffic equations for this system are

$$
\lambda_{i}=\alpha_{i}+\sum_{j \in \bar{E} \neq i} \lambda_{j} P_{j i}+\sum_{j \in E \neq i} \mu_{j} P_{j i} .
$$

These equations are solved by

$$
\begin{aligned}
\lambda_{\bar{E}} & =\left(\boldsymbol{I}-\boldsymbol{P}_{\bar{E} \bar{E}}^{\top}\right)^{-1}\left(\boldsymbol{\alpha}_{\bar{E}}+\boldsymbol{P}_{E \bar{E}}^{\top} \boldsymbol{\mu}_{E}\right), \\
\lambda_{E} & =\boldsymbol{\alpha}_{E}+\boldsymbol{P}_{E E}^{\top} \boldsymbol{\mu}_{E}+\boldsymbol{P}_{\bar{E} E}^{\top} \lambda_{\bar{E}} \\
& =\boldsymbol{\alpha}_{E}+\boldsymbol{P}_{\bar{E} E}^{\top}\left(\boldsymbol{I}-\boldsymbol{P}_{\bar{E} \bar{E}}^{\top}\right)^{-1} \boldsymbol{\alpha}_{\bar{E}}+\left(\boldsymbol{P}_{E E}^{\top}+\boldsymbol{P}_{\bar{E} E}^{\top}\left(\boldsymbol{I}-\boldsymbol{P}_{\bar{E} \bar{E}}^{\top}\right)^{-1} \boldsymbol{P}_{E \bar{E}}^{\top}\right) \boldsymbol{\mu}_{E},
\end{aligned}
$$

where $\boldsymbol{I}$ is the identity matrix and $\boldsymbol{P}^{\top}$ denotes the transpose of $\boldsymbol{P}$. Formulae similar to (1) were derived by Goodman and Massey [4], in a paper on transient Jackson networks, in which nodes $i \in E$ were unstable.

A necessary condition for stability is $\boldsymbol{\mu} \geq \lambda$. The nodes with the infinite supply of work introduce new items into the system at the following rates:

$$
\eta_{E}=\mu_{E}-\lambda_{E}
$$

It is easy to establish that $\boldsymbol{\mu}>\boldsymbol{\lambda}$ is sufficient for stability, and indeed to partially derive steady-state distributions.

Proposition 1. Assume that $\rho_{i}=\lambda_{i} / \mu_{i}<1, i=1, \ldots, I$.

(i) For nodes $i \in \bar{E}$, the joint steady-state distribution is of product form:

$$
\lim _{t \rightarrow \infty} \mathrm{P}\left\{Q_{i}(t)=n_{i}, I \in \bar{E}\right\}=\prod_{i \in \bar{E}}\left(1-\rho_{i}\right)\left(\rho_{i}\right)^{n_{i}} .
$$

(ii) For nodes $i \in E$, the marginal steady-state distribution is

$$
\lim _{t \rightarrow \infty} \mathrm{P}\left\{Q_{i}(t)=m\right\}=\left(1-\rho_{i}\right)\left(\rho_{i}\right)^{m}, \quad i \in E .
$$

(iii) The departure streams from node $i \in E$ to all other nodes $j \neq i$ are independent Poisson streams of rates $\mu_{i} P_{i j}$.

(iv) The arrival streams into node $i \in E$ from all other nodes $j \neq i$ are independent Poisson streams of rates $\lambda_{j} P_{j i}, j \in \bar{E}$ and $\mu_{j} P_{j i}, j \in E$.

The key observation for the proof of this proposition is that each of the nodes $i \in E$ works non-stop, processing items for independent and identically exponentially distributed times at rate $\mu_{i}$. Hence, departures from the nodes with the infinite supply of work consist of independent Poisson streams. Thus, the subnetwork of nodes $i \in \bar{E}$ behaves like a Jackson network with Poisson inputs. 


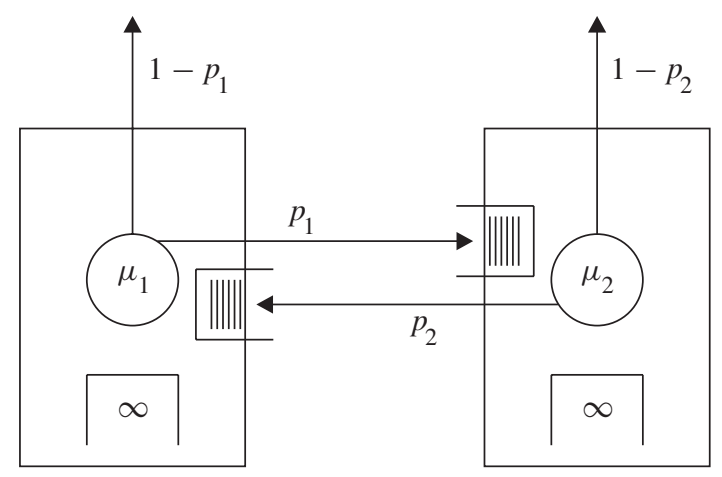

FIGURE 1: A two-node Jackson network with an infinite supply of work.

The difference between the nodes with an infinite supply of work and the standard nodes is intriguing. For the nodes with an infinite supply of work, all the input and output streams are Poisson. It is well known [7] that the streams of items moving between nodes in the Jackson part of the network, $i \in \bar{E}$, are not necessarily Poisson. (All the streams of items entering and leaving the subnetwork are Poisson, and streams between, say, node $i$ and node $j$ may be Poisson in some special cases, for example in overtake-free networks. However, in general, the stream of customers moving from node $i$ to node $j$ in a Jackson network need not be Poisson.) On the other hand, the product form, i.e. the independence of the queue lengths at different nodes when observed all at the same time, $t$, is lost for nodes $i \in E$. It is no longer true that $Q_{i}(t)$ and $Q_{j}(t), i, j \in E$, in steady state are independent. This loss of independence can be observed in the following example.

In a recent paper, Adan and Weiss [1] analyzed a two-node Jackson network with an infinite supply of work, and no exogenous input. This system is depicted in Figure 1. In Table 1, we list the values of the stationary probabilities for such a symmetric two-node Jackson network with an infinite supply of work, with $\mu_{1}=\mu_{2}, p_{1}=p_{2}=0.5$, and

TABLE 1: Stationary distribution of the infinite-supply Jackson network.

\begin{tabular}{cccccc}
\hline$n_{2}$ & $n_{1}=0$ & $n_{1}=1$ & $n_{1}=2$ & $n_{1}=3$ & $n_{1}=4$ \\
\hline 0 & 0.151946 & 0.151946 & 0.094020 & 0.050354 & 0.025752 \\
1 & 0.151946 & 0.057926 & 0.021833 & 0.009457 & 0.004471 \\
2 & 0.094020 & 0.021833 & 0.005688 & 0.001918 & 0.000802 \\
3 & 0.050354 & 0.009457 & 0.001918 & 0.000481 & 0.000161 \\
4 & 0.025752 & 0.004471 & 0.000802 & 0.000161 & 0.000040 \\
\hline
\end{tabular}

TABLE 2: Product-form joint distribution.

\begin{tabular}{lllccc}
\hline$n_{2}$ & $n_{1}=0$ & $n_{1}=1$ & $n_{1}=2$ & $n_{1}=3$ & $n_{1}=4$ \\
\hline 0 & 0.25 & 0.125 & 0.0625 & 0.03125 & 0.015625 \\
1 & 0.125 & 0.0625 & 0.03125 & 0.015625 & 0.007813 \\
2 & 0.0625 & 0.03125 & 0.015625 & 0.007813 & 0.003906 \\
3 & 0.03125 & 0.015625 & 0.007813 & 0.003906 & 0.001953 \\
4 & 0.015625 & 0.007813 & 0.003906 & 0.001953 & 0.000977 \\
\hline
\end{tabular}


$\rho_{1}=\rho_{2}=0.5$. The occupancies of the two queues are clearly not independent under this distribution. For comparison, in Table 2 we give the corresponding probabilities for a standard symmetric two-node product-form Jackson network, with $\rho=0.5$. The calculations use the results of [1].

The following intuitive thought may provide an explanation of the dependence between the two nodes in the system of Figure 1. A large number of items in node 1 indicates that there were more arrivals than departures in the recent past. Arrivals come from node 2, and departures go to node 2, so this may indicate more departures and fewer arrivals at node 2 in the recent past. Thus, observing many customers in node 1 may indicate a small number of customers in node 2 . This form of negative correlation is indeed observed in Table 1.

It is a challenging question to derive similar results in the general case.

\section{References}

[1] Adan, I. J. B. F. And Weiss, G. (2005). A two-node Jackson network with infinite supply of work. Prob. Eng. Inf. Sci. 19, 191-212.

[2] Adan, I. J. B. F. And WeIss, G. (2005). Analysis of a simple Markovian re-entrant line with infinite supply of work under the LBFS policy. To appear in Queueing Systems Theory Appl.

[3] Gans, N. And Zhou, Y. (2003). A call-routing problem with service-level constraints. Operat. Res. 51, $255-271$.

[4] Goodman, J. B. and Massey, W. A. (1984). The non-ergodic Jackson network. J. Appl. Prob. 21, 860-869.

[5] JACKSON, J. (1957). Networks of waiting lines. Operat. Res. 5, 518-521.

[6] Levy, Y. And Yechiali, U. (1975). Utilization of idle time in an M/G/1 queueing system. Manag. Sci. 22, 202-211.

[7] Kelly, F. P. (1979). Reversibility and Stochastic Networks. John Wiley, New York.

[8] Kopzon, A. And WeIss G. (2002). A push-pull queueing system. Operat. Res. Lett. 30, 351-359.

[9] WeIss, G. (2004). A fluid approach to the control of processing networks over a finite time horizon, using separated continuous linear programs, virtual infinite buffers and maximum pressure policies. Preprint, Department of Statistics, University of Haifa.

[10] WeIss, G. (2004). Stability of a simple re-entrant line with infinite supply of work - the case of exponential processing times. J. Operat. Res. Soc. Japan 47, 304-313. 